\title{
Johns Hopkins Aramco Healthcare Experience in Management Of Cancer Patient's Treatment During COVID -19 Pandemic Lockdown Of Qatif Governorate
}

\author{
Huda Alsayedahmed ${ }^{1 *}$, Nafeesa Alfaris ${ }^{2}$, Adil Alkhatti ${ }^{2}$, Samer Abushullaih ${ }^{2}$, Fuad Alghamdi ${ }^{3}$, Joshua Sharp ${ }^{2}$, Raed \\ Alsharraya $^{2}$, Salam Abughaida ${ }^{2}$, Issam Abduljaber ${ }^{2}$ and Dalal Almuhaisen ${ }^{2}$ \\ ${ }^{1}$ Department of Quality and patient safety, Johns Hopkins Aramco Healthcare, Saudi Arabia \\ ${ }^{2}$ Department of cancer institute, Johns Hopkins Aramco Healthcare, Saudi Arabia \\ ${ }^{3}$ Department of pharmacy, Johns Hopkins Aramco Healthcare, Saudi Arabia
}

*Corresponding author: Huda Alsayedahmed, PhD, Department of Quality and patient safety, Johns Hopkins Aramco Healthcare, 31911, Dhahran, Saudi Arabia

\begin{abstract}
Background: COVID-19 pandemic is a serious ongoing global public health crisis. It significantly impacted healthcare systems ability to deliver effective and timely cancer treatment. Cancer patients are at increased risk of death from corona virus. It is essential to continue the healthcare of cancer-patients who reside in a lockdown-zone. Therefore, redeployment of healthcare resources and strategies is vital.

Objective: To highlight JHAH efforts in initiating a remote cancer-therapy infusion center in a lockdown-zone located in Qatif governorate, Saudi Arabia, during March to April 2020, to ensure the continuation of quality evidence-based oncology treatment to eligible patients in a timely manner.

Methods: JHAH medical staff residing in Qatif, already under lockdown as precautionary measures, was redeployed to work in a contracted private hospital (Al Zahra General Hospital; ZGH). To provide the required treatment for JHAH cancer patient who were banned from exiting the lockdown-zone and receiving cancer-treatment at the oncology centre in Dhahran. Oncology treatment team reviewed treatment protocols for all scheduled cancer patients. Patients had the required laboratory tests in ZGH, ahead of treatment date. If the results were acceptable, treatment orders were released, and pharmacy prepared the medications for next day appointments. Medications were delivered to ZGH in Qatif governorate, where JHAH medical team administered them and provided the appropriate care.
\end{abstract}

Results: 116 JHAH cancer patients received various treatments safely as planned in ZGH. Despite an ongoing global pandemic, the care delivery efforts of the oncology team at JHAH resulted in a patient satisfaction rate above $92 \%$.

Conclusions: Our experience showed that delivering quality care for cancer patients under lockdown was feasible and successful. A well-established healthcare system with supportive leadership, multidisciplinary teamwork and a passion to provide quality and effective service to cancer patients are essential for continuity of care during a pandemic event.

Keywords: COVID-19 pandemic; Cancer-treatment; Lockdown; Precautionary measures

\section{Introduction}

Corona virus disease 2019 (COVID-19) emerged in China during the last quarter of 2019 and is responsible for the ongoing pandemic affecting people and healthcare systems worldwide. Its rapid spread amongst humans suggests that COVID-19 is a community-acquired respiratory virus (CARV) [1] that causes respiratory illness [2,3]. Patients with chronic health conditions and immunocompromised patients are at higher risk of complications from COVID [4-6]. Patients with active cancer have an increased 
risk of death compared to other comorbidities [6]. A preliminary report from Italy indicates that $18-20 \%$ of patients with COVID-19 had been diagnosed with cancer in the preceding 5 years $[7,8]$. Cancer patients were reported to have a twofold increased risk of contracting COVID-19 compared to the rest of population $[9,10]$. Nosocomial infections within the healthcare services were also observed, which stresses the importance of proper infection control [11]. A cohort study in Wuhan, China revealed that the case rate for COVID-19 in hospitalized cancer patients was 10\%, compared to $7 \%$ in healthcare workers, with higher severity and mortality in cancer patients [12]. A global political commitment to pandemic preparedness is essential. Public health measures including containment and precautionary measures have been implemented worldwide to interrupt viral transmission. Many countries applied lockdown, encouraged social distancing and cancelled mass gatherings [13]. Saudi Arabia has implemented additional restrictive measures, one of which disturbed important religious events that occur annually and affects Muslims worldwide. In 2020, Umrah and Hajj were severely restricted $[14,15]$. As COVID-19 cases increase substantially around the world, it will take longer time to control the infection. Therefore, implemented control measures will remain in place for an extended period of time [16]. Lockdown is a major challenge for healthcare systems to overcome while providing safe, timely, effective and efficient healthcare delivery. Restricting visits to hospitals or treatment centers to minimize infection risk might adversely jeopardize cancer patient's health, $[8,16]$. Therefore, a paradigm shift in healthcare services infrastructure, workforce, staffing, and care strategies are rapidly evolving worldwide [17]. The UK National Health Service (NHS) guidelines highlighted that constraint on access to operating theatres might be problematic for cancer patients requiring surgical intervention and a contingency plan is necessary to be discussed by a multidisciplinary cancer treatment team [17-21]. Cancer treatment guidelines during COVID-19 suggest switching to oral and subcutaneous therapies rather than intravenous, whenever possible [22].

Therefore, during the COVID-19 pandemic, the benefit to risk ratio of cancer treatment requires reassessment. Depending on the magnitude of process changes within a certain region, prioritization of cancer therapy is necessary to ensure safe administration. Identification of high-risk endemic areas is important to provide sufficient medical care, since early access to medical care is vital for patient survival chances [23]. Securing access to treatment for cancer patients must be a priority. To ensure that healthcare is delivered despite the constrained resources is a challenge. Methods to be achieved may include; home medication deliveries, use of continuous infusion medication pumps, remote monitoring of patients undergoing oral treatments, and utilizing laboratory testing and healthcare treatment centers closer to home [24-26]. In Saudi Arabia, several restrictive measures have been implemented, one of which involved a lockdown for all cities nationwide, few of which were subjected to whole "quarantine" when defined as an epidemic zone, e.g. Qatif Governorate (QG) in the eastern province that has no medical facility to provide oncology services. Johns Hopkins Aramco Healthcare (JHAH) Oncology Center is located in the same province at Dhahran city. While QG was under lockdown, JHAH cancer patients living there could not access Dhahran to receive their treatment. Herein, we wish to share JHAH experience to continue cancer treatment under lockdown conditions. The main objective is to highlight JHAH efforts in initiating a remote cancer therapy infusion centre in QG during the lockdown period from March to April 2020.

\section{Patients and Methods}

An approval to publish this paper was obtained from JHAH institutional review board (IRB). The Cancer Institute at JHAH identified cancer patients with active disease and under lockdown. Patient with active disease included those eligible for curative or palliative, chemotherapy, radiotherapy, surgical intervention, biological therapy, immunotherapy, hormonal therapy, and those patients with bone marrow transplant or under immunosuppressive therapy.

\section{Re-Deployment of JHAH Resources to Continue Access to Oncology Treatment for JHAH QG Cancer Population}

There are many JHAH medical staff residing in Qatif, including physicians, nurses, pharmacists, and other allied healthcare providers. Many JHAH's cancer patients resides in Qatif as well. Because of the lockdown, those patients could not access JHAH oncology services located in Dhahran. JHAH and Cancer Institute leaders re-deployed JHAH resources in QG to initiate a remote cancer-therapy infusion site, as there is no other available oncology service center in the entire $Q G$, to continue treatment for active JHAH cancer patients. Al Zahra General Hospital (ZGH) is a private hospital located in QG. JHAH leadership contracted ZGH to use part of a vacant inpatient ward to serve as an outpatient oncology clinic/ infusion center for JHAH cancer patients. The ward included four beds, an office/clinic and a storage area. JHAH used its medical staff residing in QG to work in ZGH. With one consultant haematologist/ medical oncologist, four certified oncology nurses (one of whom was an oncology nurse educator), a pharmacist, and a phlebotomist. The oncologist and nurses were assigned to care for cancer patients during the entire therapy administration process.

An agreement was made with JHAH "shipment and receiving department" to transport any clinical equipment including personal protection equipment, medications and other supplies, daily to ZGH. Medications were delivered and administered at ZGH. A secondary JHAH temporary pharmacy was opened at Al-Muwasat hospital-Qatif (MWT) for all JHAH patients, including narcotic analgesics for cancer patients. Measures were taken to ensure that patients received their controlled medications, whilst complying with the Ministry of Health (MOH) regulations. Two months supply 
of general medications were provided to avoid unnecessary patient visits to hospital during the pandemic and lockdown time.

\section{JHAH Plan for treatment of Cancer Patients in QG}

The Oncology Treatment Centre Team (OTCT) in Dhahran is a multidisciplinary team consisting of oncology physicians, nursing manager, nurse practitioners and pharmacists. They are responsible for making collaborative treatment decisions (Figure 1) and overcoming organizational and logistical challenges of providing oncology care at ZGH. Cancer patients were prioritized according to individual therapy protocol, goal of treatment, whether curative/palliative/or control. Treatment was delayed for some patients. Elective surgeries were postponed for others, to ensure patient health and safety by avoiding hospitalization during COVID-19 pandemic. When appropriate, neoadjuvant therapy was given. Patients who were to continue their treatment as planned; a pathway to organize the setting was put in place depending on the therapy type as follows:

\section{Patients under chemotherapy protocols:}

The principle of initiating the current remote cancer-therapy infusion centre in a lockdown area is based on visiting the treatment protocols planned for all affected cancer patients, and approve it by OCTC. The medications were prepared by Dhahran pharmacy then sent daily to be administrated at ZGH. A detailed process is illustrated in (Figure 1).

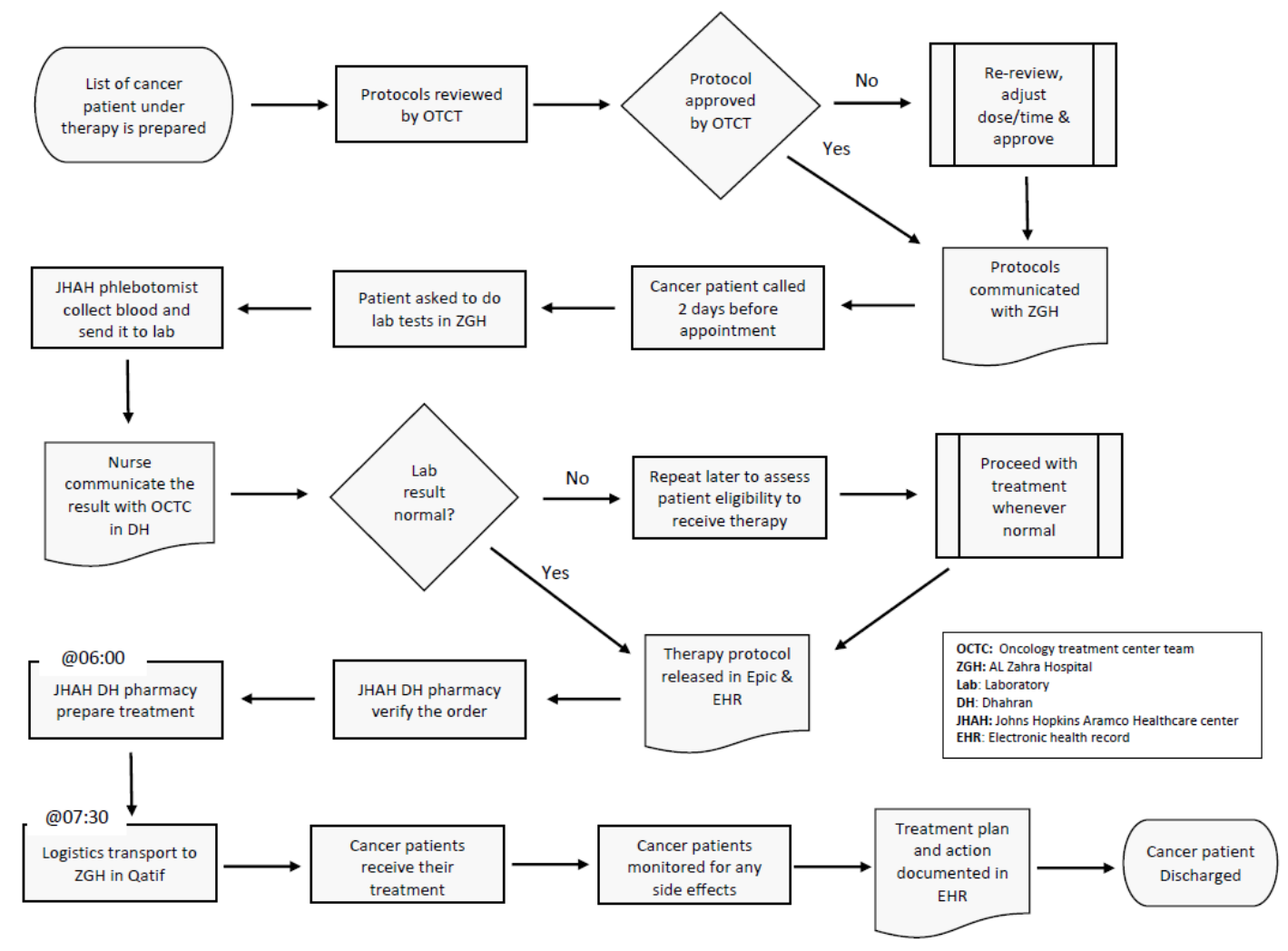

Figure 1: Process map; pathway setting for cancer therapy preparation in JHAH pharmacy in Dhahran and transporting it to ZGH in Qatif Governorate.

\section{Patients under immune, biological and hormonal therapy:}

Similar to the pathway setting of chemotherapy preparation, immune, biological and hormonal therapy protocols were prepared by JHAH pharmacy in Dhahran and transported to ZGH daily.

\section{Patients under radiotherapy protocol:}

Radiotherapy services were not available in any of QG hospitals. JHAH patients scheduled for radiotherapy were screened for COVID-19. Negative oral/nasopharyngeal swab for SARS-2 CoVi RNA was a prerequisite to obtain a special permission from the $\mathrm{MOH}$ and MOI to travel to Dhahran. They stayed in hotels quarantine for
14 days, before they could be resuming or starting radiation.

\section{Patients receiving supportive treatments Supportive:}

Medication or procedures that were also provided in ZGH including central line (CVAD) flushing, Zoledronic Acid, Factor VII and enzyme therapy administration.

\section{Patients under palliative therapy:}

Patients receiving palliative chemotherapy received their treatment as others with curative intent. They benefited from virtual clinics, all their supportive palliative medications were 
prescribed by their primary oncologist and they received it from MWT. JHAH palliative care nurse coordinator was one of the registered JHAH nurses working in ZGH; she evaluated patients and coordinated their care with Dhahran oncologists. JHAH patients who were admitted to a medical designated medical facility (MDF) in $\mathrm{QG}$, and deemed to require transfer to $\mathrm{JHAH}$, permission was obtained through MOH.

\section{Plan if any COVID-19 Symptoms Present During Treatment Period}

Visual triage was performed in a segregated waiting area, signs and symptoms of cancer patients may experience therapy related side effects similar to COVID-19. In the event that a patient developed or presented with COVID-19 symptoms such as; fever, cough, sore throat or difficulty in breathing, they would be redirected for appropriate viral swabbing and their treatment postponed until a negative result was produced. Physical distancing measures were implemented by spacing out seats and limiting visitors in the treatment areas, and if a high-risk case identified, MOH was notified.

\section{Results}

A total of $116 \mathrm{JHAH}$ cancer patients with solid and haematological malignancies on active treatment were served at ZGH during the lockdown period in QG between March to April 2020. This included 30 patients who continued their chemotherapy as planned, 33 patients received biological immunotherapies, 15 patients received hormonal treatment, and 22 patients received supportive treatments. Five patients who were on palliative therapy received care as well. Eleven patients were undergoing radiotherapy, were transferred to JHAH oncology centre in Dhahran and treated. (Figure 2) In addition, a total of 140 blood tests were performed in ZGH laboratory including repeated abnormal tests results. One patient had a Lumbar Puncture for administration of intrathecal chemotherapy.

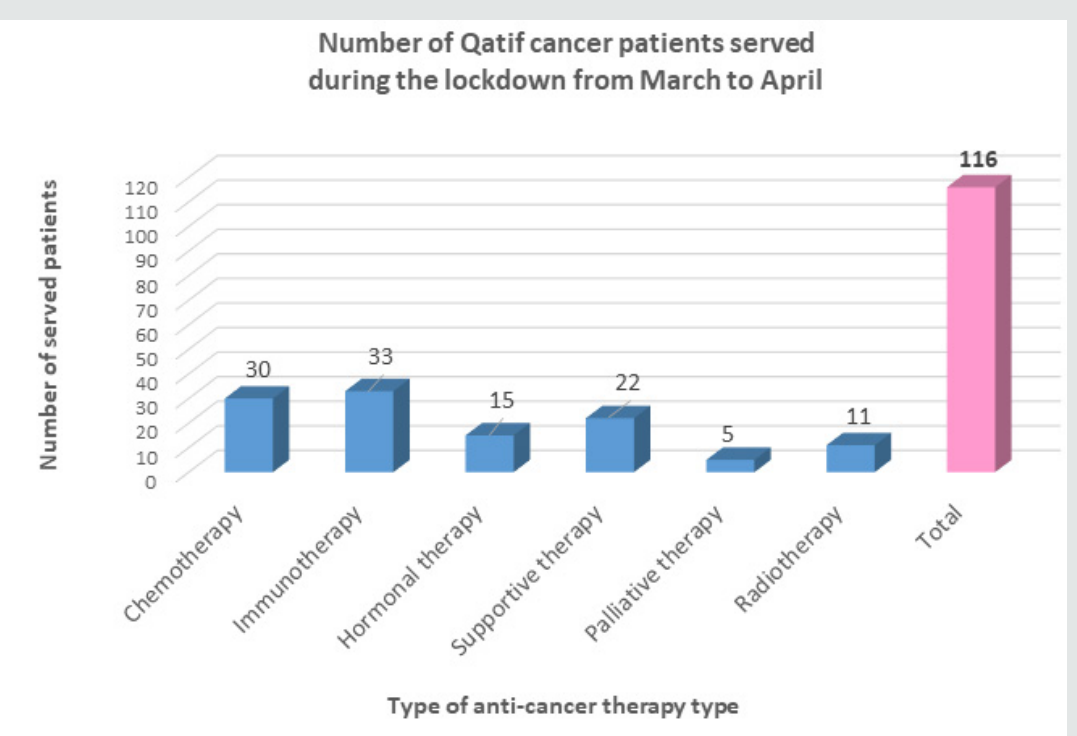

Figure 2: Total number of Qatif cancer care patients who received their corresponding therapy during the lockdown period during March and April.

\section{Patients who experienced a reaction/ side effects and/ or COVID-19 related symptom}

One patient developed an acute infusion reaction while receiving chemotherapy (Oxaliplatin). She was treated, stabilized and then referred to MWT for further care. Two patients developed neutropenia. One patient was given filgrastim (G-CSF) and therapy was delayed for one week. when he recovered and received treatment. The other patient therapy was delayed by a week. He recovered spontaneously and was given the scheduled chemotherapy. Fortunately, during the lockdown period, there was no reported COVID-19 case amongst our patients. However, one JHAH staff nurse who had flu-like symptoms, immediately ceased work, had viral testing which was negative for COVID-19.

\section{Indicators of optimal performance}

The patient's satisfaction rate remained above 92\%, despite challenges faced during lockdown period. (Figure 3) 


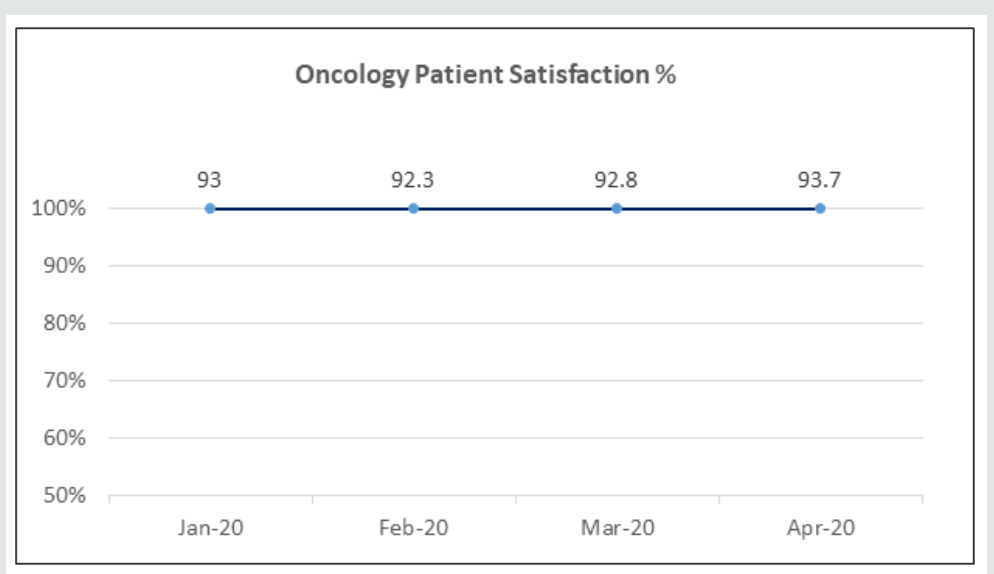

Figure 3: Oncology patient satisfaction rate, kept above the $90 \%$ during the lockdown period during March and April.

\section{Discussion}

During the COVID-19 lockdown period, JHAH revisited the process of treating cancer patients. Patients who were locked down in QG, and whose therapy was judged to have to continue during the pandemic were identified. Personnel who resides in the area were assigned to deliver the care at a local hospital; ZGH. Equipment and supplies including emergency medications were transported from JHAH. Chemotherapy, biological, hormonal and adjunct therapies were administered. Individual patient treatment protocols were prepared in JHAH Dhahran-pharmacy and transferred daily to Qatif. Some patients were initially apprehensive about coming to an unfamiliar care setting and cared for by different environment. The risk of COVID-19 transmission was another worry. Initial apprehension abated over time. Dealing with emergencies, especially infusion reactions, was a patient safety concern. While emergency medications and services were available in $\mathrm{ZGH}$, the clinical setting and availability of immediate help was an identified risk. Evaluation of response to non-adjuvant therapy was largely limited to clinical evaluation. At least one patient has disease progression. As patients became comfortable with the setting, they appreciated its proximity to where they lived. This decreased commuting time and effort.

Reported approaches to cancer treatment during the COVID-19 epidemic included delaying therapy whenever possible and administering therapy only when necessary at a center in the lockdown area. These approaches were reported from China and Italy, which suffered the first two COVID-19 major epidemics [2740].

JHAH experience was unique. Treatment was provided in a lockdown area with no medical facilities able to provide oncology services. Organizational challenges were encountered. These included leading a team during a crisis and coping with a drastically changed work environment, complicated by lockdown measures. Staff shortage secondary to QG lockdown, staff trapped on vacation unable to return to the country because of dismissing flights and the MOH mandated medical leave for staff with certain chronic medical conditions and pregnancy. Moreover, disruption of supply chain could result in medications, personal protection equipment and blood product shortages. JHAH management, in collaboration with COVID-19 task force, addressed human resources (HR) and supply chain related issues ensuring that medications and equipment were adequately stocked. Faced with these challenges, JHAH oncology institute leadership increased working hours to 12 hours per shift to accommodate more patients at Dhahran. At the same time, resources were allocated for operating a secondary remote clinic/infusion center.

Logistical obstacles including transportation of treatments, medical equipment and other materials to $Q G$ required permission from the Ministry of Interior (MOI) that was enforcing the lockdown. Likewise, JHAH personnel required special permission from MOI to travel to QG. At one point, QG was divided into 4 zones with restricted movement between them. Some patients and staff required permission to move to $\mathrm{ZGH}$ and to travel in the same zone during nationwide curfew. Certain medications, with short stability after preparation, were prepared at the last minute and transported immediately. A breast cancer patient who required a breast biopsy, which could only be performed at JHAH in Dhahran, required emergency logistic arrangements to transfer the patient. This was done and the patient therapy was initiated. Clinical challenges faced while treating patients in ZGH included staff education. While all four JHAH nurses were previously registered through the Oncology Nursing Society (ONS), they have not administered chemotherapy recently. Chemotherapy-privileged nurses provided a virtual refresher-training course from Dhahran. Neoadjuvant chemotherapy was given to several breast and rectal cancer patients at both JHAH cancer institute and ZGH to mitigate the delay in surgical treatment, since elective surgeries were put on hold. Since there is no radiation facility in Qatif, patients needed permission to exit from the lockdown, which required a long time to obtain. 
COVID-19 posed a significant challenge to the global medical community. The ongoing pandemic is alerting leaders of healthcare systems to be continuously prepared for such crisis. This would include having sufficient resources available at all times for unforeseen public health challenges. In this study, JHAH put together maximum efforts to guarantee continuing cancer treatment with efficient healthcare services for patients affected by lockdown, which were successfully achieved.

\section{Conclusions}

Access to care and access to treatment during the COVID-19 crisis, especially for high-risk patients, such as cancer patients, is a huge challenge internationally. Assessment of the relative risks and benefits of continuing, versus delaying anti-cancer therapies during a pandemic is fundamental to continue safe delivery of cancer-care. Efficient redeployment of a healthcare infrastructure and resources can contribute to better cancer outcomes. Despite the challenges, the JHAH cancer institute has shown that with concerted efforts, it turned the lockdown to an advantage by assigning medical staff residing in the area, and collaborated with a local hospital to establish a temporary "satellite cancer treatment center". This enabled JHAH to continue treating more than 100 cancer patients over a period of about 7 weeks. Such process is necessary to consider as a backup plan in case of future catastrophe. In addition, it is worthy to deliberate a well-organized, remote cancertreatment center for localities far away from Dhahran to decrease the hardship of patients by serving them close to where they live.

\section{References}

1. von Lilienfeld-Toal M, Vehreschild JJ, Cornely O, Pagano L, Compagno F, et al. (2020) Frequently asked questions regarding SARS-CoV-2 in cancer patients-recommendations for clinicians caring for patients with malignant diseases. Leukemia 34(6): 1487-1494.

2. SinghalT (2020) A Review of Coronavirus Disease-2019 (COVID-19). Indian J Pediatr 87(4): 281-286.

3. Mehta P, McAuley DF, Brown M, Sanchez E, Tattersall RS, et al. (2020) COVID-19: consider cytokine storm syndromes and immunosuppression. Lancet 395(10229): 1033-1034.

4. Zhou F, Yu T, Du R, Fan G, Liu Y, et al. (2020) Clinical course and risk factors for mortality of adult in patients with COVID-19 in Wuhan, China: a retrospective cohort study. Lancet 395(10229):1054-1062.

5. Xia Y, Jin R, Zhao J, Li W, Shen H, et al. (2020) Risk of COVID-19 for patients with cancer. Lancet Oncol 21(4): e180.

6. Liang W, Guan W, Chen R, Wang W, Li J, Cancer patients in SARS-CoV-2 infection: a nationwide analysis in China. Lancet Oncol 2020; 21(3): 335-337.

7. Yang G, Zhang H, Yang Y(2020) Challenges and Countermeasures of Integrative Cancer Therapy in the Epidemic of COVID-19. Integrative cancer therapies 19: 1534735420912811.

8. Spicer J, Chamberlain C, Papa S (2020) Provision of cancer care during the COVID-19 pandemic. Nat Rev Clin Oncol 17: 329-331.

9. Al-Shamsi HO, Alhazzani W, Alhuraiji A, Coomes EA, Chemaly RF, et al. (2020) A Practical Approach to the Management of Cancer Patients During the Novel Coronavirus Disease 2019 (COVID-19) Pandemic: An
International Collaborative Group. The oncologist 25(6): e936-e945

10. Coomes EA, Al-Shamsi HO, Meyers BM, Alhazzani W, Alhuraiji A, Chemaly RF, et al. (2020) Authors Reply: How to manage febrile neutropenia during the COVID pandemic? In response to, "A Practical Approach to the Management of Cancer Patients During the Novel Coronavirus Disease 2019 (COVID-19) Pandemic". Oncologist 25: 1-2.

11. Yi-Chi W, Ching-Sung C, Yu-Jiun C (2020) the outbreak of COVID-19: An overview. J Chin Med Assoc 83(3): 217-220.

12. He W, Chen L, Chen L, Yuan G, Fang Y, et al. (2020) COVID-19 in persons with hematological cancers. Leukemia 34: 1637-1645.

13. Kickbusch I, Leung G (2020) Response to the emerging novel coronavirus outbreak. BMJ 368: m406.

14. Ebrahim SH, Memish ZA (2020) Saudi Arabia`s measures to curb the COVID-19 outbreak: temporary suspension of the Umrah pilgrimage. J Travel Med 27(3): taaa029.

15. Memish ZA, Steffen R, White P, Dar O, Azhar EI, et al. (2019) Mass gatherings medicine: public health issues arising from mass gathering religious and sporting events. Lancet 393(10185): 2073-2084.

16. Kolifarhood GA, Ghaali M, Saadati HM, Taherpour N Rahimi S, et al. (2020) Epidemiological and Clinical Aspects of COVID-19; a Narrative Review. Arch Acad Emerg Med 8(1): e41.

17. (2020) Clinical guide for the management of non-coronavirus patients requiring acute treatment. NHS England

18. Søreide K, Hallet J, Matthews JB, Schnitzbauer AA, Line PD, et al. (2020) Immediate and long-term impact of the COVID-19 pandemic on delivery of surgical services. The British journal of surgery pp.

19. Martí C, Sánchez-Méndez JI (2020) Neoadjuvant endocrine therapy for luminal breast cancer treatment: a first-choice alternative in times of crisis such as the COVID-19 pandemic. Ecancermedicalscience 14: pp. 1027.

20. Kumar S, Chmura S, Robinson C, Lin SH, Gadgeel SM, et al. (2020) Alternative Multidisciplinary Management Options for Locally Advanced Non-Small Cell Lung Cancer During the COVID-19 Global Pandemic. J Thorac Oncol 15(7): 1137-1146.

21. Williams VM, Kahn JM, Harkenrider MM, Chino J, Chen J, et al. (2020) COVID-19 impact on timing of brachytherapy treatment and strategies for risk mitigation. Brachytherapy 19(4): 401-411.

22. Burki TK (2020) Cancer guidelines during the COVID-19 pandemic. Lancet Oncol 21(5): 629-630.

23. Khafaiea MA, Rahim F (2020) Cross-Country Comparison of Case Fatality Rates of COVID-19/SARS-COV-2. Osong Public Health Res Perspect 11(2): 74-80.

24. (202) Cancer Care During the COVID-19 Pandemic: An ESMO Guide for Patients. European Society for Medical Oncology (ESMO)

25.(2020) the UK National Institute of Health and Care Excellence. COVID-19 rapid guideline: delivery of systemic anticancer treatments.

26. Mei H, Dong X, Wang Y, Tang L, Hu Y, et al. (2020) Managing patients with cancer during the COVID-19 pandemic: frontline experience from Wuhan. The Lancet Oncology 21(5): 634-636.

27. Lambertini M, Toss A, Passaro A, Criscitiello C, Cremolini C, Cardone C, et al. (2020) Cancer care during the spread of corona virus disease 2019 (COVID-19) in Italy: Young oncologists' perspective. ESMO Open 5(2): e000759.

28. Balduzzi A, Brivio E, Rovelli A, Rizzari C, Gasperini S, et al. (2020) Lessons after the early management of the COVID-19 outbreak in a pediatric transplant and hemato-oncology center embedded within a COVID-19 dedicated hospital in Lombardia, Italy. Estote parati. Bone Marrow Transplant 1-6. 
29. Indini A, Aschele C, Cavanna L, Clerico M, Daniele B, et al. (2020) Reorganisation of medical oncology departments during the novel corona virus disease-19 pandemic: a nationwide Italian survey. Eur J Cancer 132: 17-23.

30. Trapani D, Marra A, Curigliano G (2020) the experience on coronavirus disease 2019 and cancer from an oncology hub institution in Milan Lombardy Region. European Journal of Cancer 132: 199-206.

31. Wang Z, Wang J, He J (2020) Active and Effective Measures for the Care of Patients With Cancer During the COVID-19 Spread in China. JAMA Oncol 6(5): 631-632.

32. van de Haar J, Hoes LR, Coles CE, Seamon K, Fröhling S, et al. (2020) Caring for patients with cancer in the COVID-19 era. Nat Med 26(5): 665671.

33. Zhang L, Zheng Z, Hu G, Yuan X (2020) Prevention and control measure to avoid cross infection during radiotherapy in coronavirus disease 2019 (COVID-19) epidemic in Wuhan, China. Radiotherapy and Oncology 149: 104-106.

34. Tan J, Yang C (2020) Prevention and control strategies for the diagnosis and treatment of cancer patients during the COVID-19 pandemic. Br J Cancer 123: 5-6.
35. Omarini C, Maur M, Luppi G, Narni F, Luppi M, et al. (2020) Cancer treatment during the coronavirus disease 2019 pandemic: Do not postpone, do it! European journal of cancer 133: 29-32.

36. Vrdoljak E, Sullivan R, Lawler M. Cancer and coronavirus disease 2019; how do we manage cancer optimally through a public health crisis? European journal of cancer 132: 98-99.

37. Curigliano G, Cardoso MJ, Poortmans P, Gentilini O, Pravettoni G, (2020) Recommendations for triage, prioritization and treatment of breast cancer patients during the COVID-19 pandemic. Breast 52: 8-16.

38. Kattan C, Badreddine H, Rassy E, Kourie HR, Kattan J, et al. (2020) The impact of the coronavirus pandemic on the management of cancer patients in Lebanon: a single institutional experience. Future oncology 16(17):1157-1160.

39. Ueda M, Martins R, Hendry P, Terry McDonnell, Jennie R Crews, et al. (2020) Managing Cancer Care During COVID-19 Pandemic: Agility and Collaboration toward a Common Goal. J Natl Compr Canc Netw 18(4): $1-4$.

40. The ASCO Post. Practical Approach to Management of Patients With Cancer During the COVID-19 Pandemic: An International Collaborative Group Statement 2020.

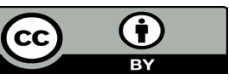

This work is licensed under Creative Commons Attribution 4.0 License

To Submit Your Article Click Here: Submit Article

DOI: $10.32474 /$ OAJOM.2020.03.000174

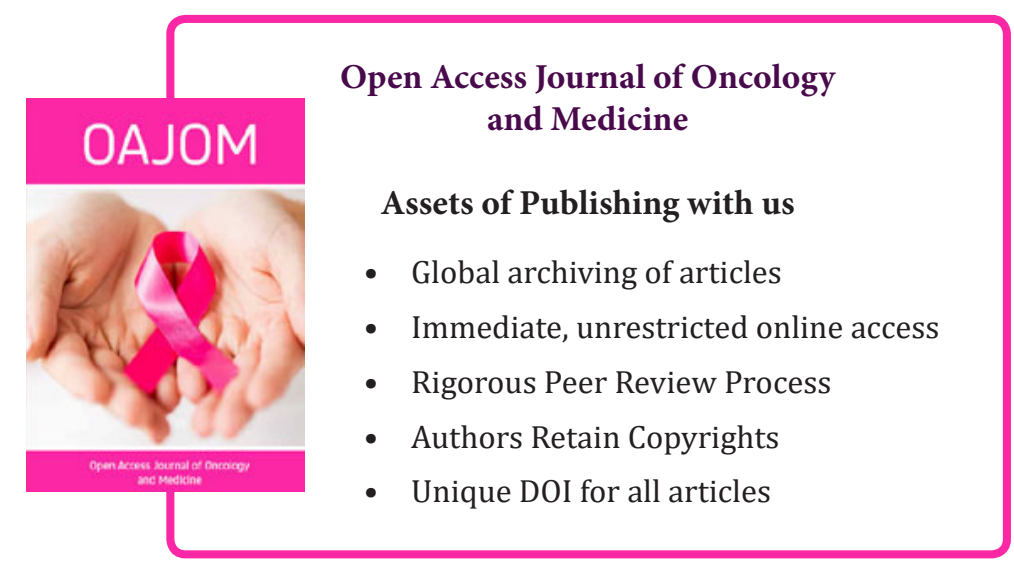

